\title{
Unconscious processing of dichoptically masked words
}

\author{
ANTHONY G. GREENWALD, MARK R. KLINGER, and THOMAS J. LIU \\ University of Washington, Seattle, Washington
}

\begin{abstract}
In three experiments, the subjects' task was to decide whether each of a series of words connoted something good (e.g., fame, comedy, rescue) or bad (stress, detest, malaria). One-half second before the presentation of each such target word, an evaluatively polarized priming word was presented briefly to the nondominant eye and was masked dichoptically by either the rapidly following (Experiment 1) or simultaneous (Experiments 2 and 3 ) presentation of a random letterfragment pattern to the dominant eye. (The effectiveness of the masking procedure was demonstrated by the subjects' inability to discriminate the left vs. right position of a test series of words.) In all experiments, significant masked priming effects were obtained; evaluative decisions to congruent masked prime-target combinations (such as a positive masked prime followed by a positive target) were significantly faster than those to incongruent (e.g., negative prime/positive target) or noncongruent (e.g., neutral prime/positive target) combinations. Also, in two of the three experiments, when subjects were at chance accuracy in discriminating word position, their position judgments were nevertheless significantly influenced by the irrelevant semantic content (LEFT vs. RIGHT) of the masked position-varying words. The series of experiments demonstrated that two very different tasks-speeded judgment of evaluative meaning and nonspeeded judgment of word position-yielded statistically significant and replicable influences of the semantic content of apparently undetectable words. Coupled with previous research by others using the lexical decision task, these findings converge in establishing the reliability of the empirical phenomenon of semantic processing of words that are rendered undetectable by dichoptic pattern masking.
\end{abstract}

Historically, a severe problem with psychological uses of the word unconscious has been the lack of consensus as to what research operations justify that word's use. Nevertheless, in recent years two categories of wellestablished phenomena have been increasingly assimilated to the unconscious label: (1) preattentive processing encompasses stimulus analyses that occur despite inattention to, and lack of memory for, auditory or visual inputs in selective attention experiments (e.g., Moray, 1970; Rock \& Gutman, 1981); and (2) procedural knowledge designates motor or perceptual skills that are performed with little or no attention demand and, correspondingly, little or no memory for details of individual performances (e.g., Anderson, 1982). These two sets of phenomena can be understood as varieties of attentionless processingprocessing of information that is potentially attended, but is not attended, because attention is directed elsewhere.

This article focuses on a more controversial category of putatively unconscious phenomena-phenomena that may be labeled detectionless processing, or processing of information from stimuli that cannot be detected and

This research was supported by NSF Grant BNS-82-17006 and by NIMH Grant MH-41328. The authors thank Thomas H. Carr, Matthew Hugh Erdelyi, Russell H. Fazio, Philip M. Merikle, and anonymous reviewers for comments on an earlier draft. Requests for reprints should be addressed to Anthony G. Greenwald, Department of Psychology, NI-25, University of Washington, Seattle, WA 98195. are therefore not potentially within attentional focus. ${ }^{1}$ Most claimed findings of detectionless processing have been controversial, either because they have been difficult to replicate or because their procedures have not conclusively established the nondetectability of critical stimuli. This controversiality can be seen, for example, in authoritative reviews of research on the topics of perceptual defense (Erdelyi, 1974; Eriksen, 1958) and subliminal influence (Moore, 1982).

\section{UNCONSCIOUS PRIMING OF LEXICAL DECISIONS}

Against the background of controversy about detectionless processing, one recently reported finding offers some potential for providing a replicable result worthy of that label. This finding has been described, in the three studies in which it has been obtained, as "unconscious perception" (Marcel, 1983), "lexical access without awareness" (Fowler, Wolford, Slade, \& Tassinary, 1981), and "automatic semantic activation"' (Balota, 1983). All three of these studies used a procedure in which each trial of a lexical decision task-judging whether or not a letter string was a word-was preceded by presentation of another letter string that was backward-masked for the purpose of preventing its detection. The masking technique used in all three studies, backward dichoptic pattern masking, involved presenting a to-be-masked letter string to the non- 
dominant eye, followed rapidly by a pattern mask (e.g., an array of randomly oriented letter fragments) presented to the dominant eye. The interval between letter string and mask was adjusted for each subject to a value at which the subject failed to discriminate trials on which a letter string preceded the mask from those on which no letter string was presented.

Previous research has shown that positive lexical decisions are facilitated by preceding the to-be-judged word with a semantically associated word (Meyer \& Schvaneveldt, 1971). Marcel (1983, Experiment 4) showed that this priming of lexical decisions was as strong when priming words were made nondetectable by backward dichoptic pattern masking as when they were fully visible. Both Fowler et al. (1981) and Balota (1983) replicated Marcel's finding. Importantly, Marcel reported that the masked priming effect was not obtained with a nondichoptic (monocular) energy masking procedure, in which the letter string and mask were presented in rapid succession to the same eye. In replicating Marcel's result, Fowler et al. and Balota also used the dichoptic pattern masking procedure.

Three independent replications of essentially the same finding make a strong case for its validity. Nevertheless, two criticisms of these studies have been raised. First, Holender (1986, following the lead of Purcell, Stewart, \& Stanovich, 1983) suggested that the use of different illumination patterns in threshold-setting and masked priming tests could have led to the selection of too-long thresholds; these, in turn, could have allowed masked priming stimuli to be visible. Second, Cheesman and Merikle (1985) observed that the threshold-setting procedures of the three studies may have located subjective, rather than objective, thresholds. In other words, subjects may have reported that they saw nothing even when stimuli were partly visible. Although it is not clear that the illumination-pattern and subjective-threshold criticisms actually apply to the studies by Marcel (1983), Fowler et al. (1981), and Balota (1983), still the criticisms succeeded in raising doubt as to whether these studies demonstrated detectionless processing. (These two criticisms are considered in more detail in the General Discussion section.)

\section{PRESENT PROCEDURES}

In seeking a more conclusive verdict on detectionless processing of word meaning, the present research used a novel procedure to establish perceptual thresholds for word stimuli, and used two new tests for detectionless processing of word meaning.

\section{Threshold-Testing Task}

In the present research, threshold stimulus-presentation conditions were ones under which the subjects performed with chance accuracy in judging whether briefly flashed test words were presented just right or just left of a fixation point. This task avoided a problem for which the task of judging word presence or absence (used in some previous detectionless processing studies) had been criticized. Merikle (1982; see also Cheesman \& Merikle, 1985) argued that subjects might be reluctant to say "present" in response to a partially visible word and therefore might give responses that falsely indicated that they were at or below threshold. With the position-judgment task, subjects should not be similarly reticent to report one of the two response alternatives (i.e., left or right). ${ }^{2}$ An additional important reason for using the position-judgment task was that it provided the basis for one of the study's two tests of detectionless processing.

\section{Evaluative Decision Task}

The subjects' major task in the present research was to judge whether each of a series of presented (target) words was evaluatively good or bad in meaning. Because evaluation is a major component of word meaning (Osgood, Suci, \& Tannenbaum, 1958), evaluatively polarized preliminary words should produce priming-that is, facilitation of evaluative decisions by primes that are evaluatively congruent with targets, and interference by evaluatively incongruent primes. Such priming effects have been demonstrated by Fazio, Sanbonmatsu, Powell, and Kardes (1986). A virtue of the evaluative decision task is that data useful for hypothesis tests are obtained from all trials, whereas useful data are obtained from only about one-third of trials with the lexical decision task. (In the lexical decision task, trials that have nonword primes and/or nonword targets are not used in estimating priming effects.)

\section{Detectionless Processing Test on \\ Threshold Testing Trials}

Threshold tests required the subjects to judge whether a word stimulus was located just left or just right of a fixation point. To make extra use of these trials, the word stimuli used were LEFT and RIGHT, and these were presented so that word content agreed with actual word position on $50 \%$ of the trials and conflicted on the remaining $50 \%$. This made it possible to determine whether semantic content could influence position judgments at the same time that the subjects' overall performance in judging actual word position was at chance.

\section{EXPERIMENT 1}

\section{Method}

Subjects. The subjects were 20 volunteers, male and female, who participated in partial fulfillment of a requirement of their introductory psychology course. Only persons with normal or correctedto-normal vision and fluency in English were allowed to participate. Data for 4 additional volunteers were not included in analyses, 3 because of apparatus failures and 1 because of astigmatism that prevented reading the dichoptically presented words.

Apparatus. Stimuli were presented via a Gerbrands three-field tachistoscope, which was modified by adding a permanent fixation point in the form of a low-luminance light-emitting diode as a fourth field. Additionally, the fields for presentation of prime and target stimuli had automatic card changers, and those for prime and mask 
stimuli contained polarizing filters. Complementary polarizing filters in the left and right openings of the eyepiece were oriented so that the prime field could be presented exclusively to the subject's nondominant eye and the mask field exclusively to the dominant eye. The target field was visible to both eyes. With polarizing filters in place, measured luminances of the prime, mask, and target fields were, respectively, $13.2,13.4$, and $16.6 \mathrm{~cd} / \mathrm{m}^{2}$. A footswitch, operated by the subject, initiated a $0.5-\mathrm{sec}$ delay before the tachistoscope sequence for each trial started. A small box, on the top of which were two pushbutton switches mounted $14.3 \mathrm{~cm}$ apart, was used to indicate the subject's responses, made with the left and right forefingers. The response on each trial (left or right) and its latency in milliseconds were recorded by a microcomputer.

Stimuli. The masking stimulus was a rectangular $(2.54 \times 6.67 \mathrm{~cm})$ collection of randomly oriented letter fragments, presented subtending $1.85^{\circ}$ (vertical) $\times 4.85^{\circ}$ of visual angle, centered on the fixation point. (The same mask was used in all of the present experiments.) Priming stimuli were 18 high-frequency words, including 6 evaluatively positive words (happy, joy, peace, love, excellent, pleasant), 6 evaluatively negative words (evil, grief, sad, gloom, ugly, horrid), and 6 neutral words (contents, chair, ink, door, green, street). Most of these words were selected from 399 words for which pleasantness norms were obtained in a study by Bellezza, Greenwald, and Banaji (1986). On a 1-5 pleasantness scale, for which 1 was defined as very unpleasant and 5 was defined as very pleasant, the unpleasant, neutral, and pleasant priming words were within the ranges $1.0-1.6,2.5-3.5$, and 4.4-5.0, respectively. Target stimuli were 72 evaluatively negative and 72 evaluatively positive words, selected from the ranges $1.0-2.0$ and $4.0-5.0$, respectively, of the Bellezza et al. norms. Prime and target words were presented centrally in their respective fields, and were printed in capital letters that were $0.41 \mathrm{~cm}$ high and $0.23 \mathrm{~cm}$ wide. Words subtended $0.30^{\circ}$ (vertical) $\times 0.53^{\circ}$ (three letters) to $1.64^{\circ}$ (nine letters) of visual angle.

Eye dominance determination. The subject was asked to point at a switch on the laboratory wall, close each eye in turn, and report whether the switch was still aligned with the pointing finger. (Alignment is lost when the dominant eye is closed.) The procedure was repeated with a different target to confirm the dominance determination. The tachistoscope's eyepiece filters were then arranged so that the prime field and the mask field would be presented to the subject's nondominant and dominant eye, respectively.

Threshold determination (position-discrimination task). Threshold determination started after a 10 -min dark adaptation period. Test stimuli consisted of a random series of the words RIGHT and LEFT, positioned randomly either just left or just right of the central fixation point. (These test words were fully within foveal vision when the subject was looking at the fixation point.) The subject was asked to press the left or right button, depending only on whether the test stimulus was seen to the left or right of center, and ignoring the semantic content of the word, which was independent of its position (as previously described, this variation of word content provided a second test of detectionless processing). Following the subject's press of the footswitch, each trial consisted of a sequence of (1) a test stimulus presented for $10 \mathrm{msec}$ to the nondominant eye (in the prime field); (2) a dark interval that varied during the course of testing; (3) the pattern mask for $30 \mathrm{msec}$ to the dominant eye; (4) a second dark interval, the duration of which was computed by subtracting the sum of the first three intervals from $500 \mathrm{msec}$; and (5) the target field, containing the words PLEASE CONTINUE, presented to both eyes for $2 \mathrm{sec}$ (see Figure 1). (The temporal pattern of illumination levels on these trials was identical to that on subsequent masked priming test trials.) The subject was instructed to respond on each trial by pressing the button on the side on which the test stimulus was seen, and to guess if uncertain. After responding, the subject waited to hear the card changer operate before starting the next trial with a footswitch press.

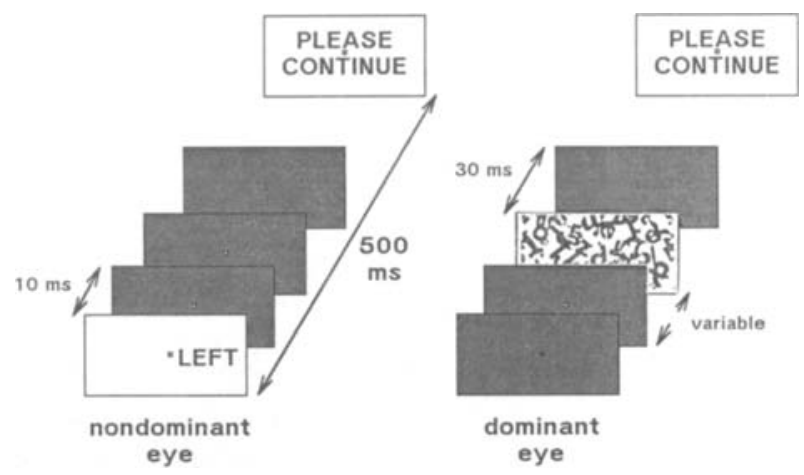

Figure 1. Sequence of events for threshold-setting trials of Experiment 1 . The subject's task was to judge whether the word in the first frame to the nondominant eye was to the left or right of the continually present fixation point. The pattern mask shown in the third field for the dominant eye is the central portion of the one that was used.

For the first 6 trials, a dark interval of $200 \mathrm{msec}$ between test stimulus offset and mask onset was used, and this was long enough to permit all subjects to see the position of the test stimulus clearly. This interval was reduced, in successive blocks of 6 trials, to 100 , $70,50,30,20,15$, and $12 \mathrm{msec}$, then reduced further in 1 -msec decrements until the subject produced at least four errors in one of these blocks of 6 trials. At that point, 24 more trials were conducted without changing the test-stimulus-to-mask interval. A testto-mask interval was considered usable if the subject produced at least 12 errors in the final 24 trials. (Otherwise, the dark interval was further reduced and testing was continued.) This procedure required each subject to make at least 16 position-judgment errors in 30 trials (i.e., at least $53 \%$ errors, compared with the chance expected value of $50 \%$ ). ${ }^{3}$ The resulting threshold stimulus onset asynchronies (SOAs; intervals between test stimulus onset and mask onset) ranged from 15 to $25 \mathrm{msec}$ across the 20 subjects.

Evaluative decision task-Masked priming trials. After the threshold-setting phase, the subject was told that on each trial there would be an initial flash, following which an easily visible word would be presented. The subject's task was to judge the word on each trial as being evaluatively good or bad in meaning, by pressing the right or left response button (respectively) as rapidly as possible. Four practice masked priming trials were used to acquaint the subject with this task, following which another 72 trials were presented. The subject started each trial by pressing the footswitch. Six types of trials were constructed by combining the three types of primes (positive, neutral, and negative) with the two types of targets (positive and negative). There were 12 trials of each of these six types in the series of 72 . The order of types within the 72 trials was random, with the restriction that each of the 36 possible sequences of two (of the six) trial types occurred once in the first 36 and once in the last 36 trials. The prime, first dark interval, mask, and second dark interval were presented for the durations established during the threshold-determination series. After the second dark interval (i.e., $500 \mathrm{msec}$ after onset of the prime), the target word was presented to both eyes for $2 \mathrm{sec}$, during which the subject responded. The sequence of events on a masked priming trial is diagrammed in Figure 2.

Visible priming trials. Visible priming trials consisted also of prime, mask, and target stimuli, separated by dark intervals: however, the prime was presented to both eyes and its duration was increased to $210 \mathrm{msec}$. A $10-\mathrm{msec}$ dark interval followed the prime, after which the mask was presented for $30 \mathrm{msec}$ to both eyes, followed by a 250 -msec dark interval, and then presentation of the target for $2 \mathrm{sec}$. (This preserved the prime-target onset interval at 


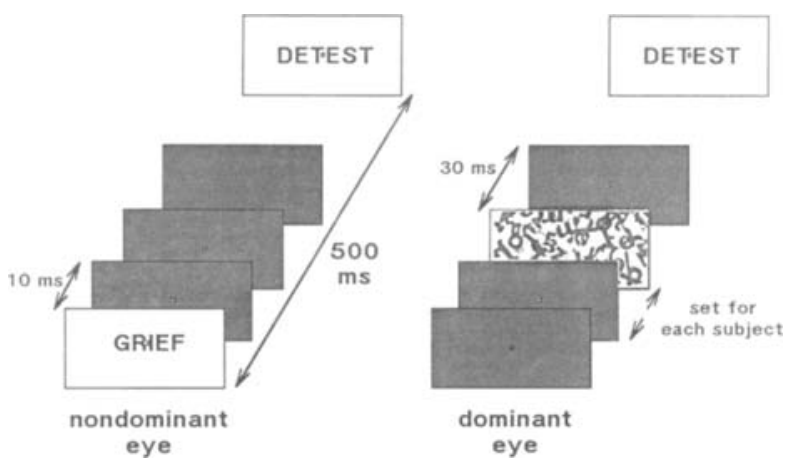

Figure 2. Sequence of events on a masked priming trial of Experiment 1. The subject's task was to judge whether the word presented in the final frame to both eyes was evaluatively positive or negative in meaning.

$500 \mathrm{msec}$, the same as for the masked priming condition.) The subject was instructed to look at the initial (prime) word, but not to respond to it. Like the masked priming condition, the visible priming condition started with 4 unanalyzed practice trials, followed by 72 experimental trials. The sequence of priming stimuli was the same as that used in the masked condition; however, a different set of target words was used.

The masked condition was always presented first, so that the subjects would not be alerted to the inclusion of priming words in the masked condition's trial-beginning flash. At the conclusion of the experiment, the subjects were interviewed informally to determine whether they had been able to detect words in the initial flashes of masked trials. All subjects reported that they had not been aware of such words. (This was not intended as a means of confirming the nondetectability of masked primes; however, concern would have been raised if the subjects had reported awareness of words.)

\section{Results}

Errors occurred on $7.5 \%$ of trials of the evaluative decision task, and were more frequent on visible priming trials $(11.7 \%)$ than on masked priming trials $(3.4 \%)$. The latency analyses reported include data from error trials as well as correct trials. ${ }^{4}$ The obtained data included some latencies that were too short to have been responsive to the stimuli, as well as some very long latencies that were produced when card-changer malfunctions caused the target not to be presented. Examination of the distribution of latencies indicated that the variance-inflating effects of these outliers could be eliminated by deleting 34 trials ( $1.2 \%$ of all trials) on which latencies were shorter than $400 \mathrm{msec}$ or longer than $1,600 \mathrm{msec}$.

Masked and visible priming effects. Figure 3 presents mean reaction-time data for each of the six trial types in both the masked and visible priming conditions. Obtained priming effects consisted more of facilitation by evaluative congruence of prime and target than of interference by evaluative incongruence. (These priming effects of positive and negative primes were assessed by using the neutral-prime conditions as a baseline; only the facilitative priming effects were statistically significant.) Overall tests of priming assessed the significance of the average latency differences between congruent and incongruent priming conditions. This overall test con- firmed highly significant priming effects in both the masked priming condition [799 - $725=74 \mathrm{msec}$, SEM $=13.6 \mathrm{msec} ; F(1,19)=30.10, p<.001]$ and the visible priming condition $[779-723=56 \mathrm{msec}, S E M=$ $17.2 \mathrm{msec} ; F(1,19)=10.67, p<.011$. In sum, priming effects were clearly demonstrated in both the masked and visible priming conditions.

Masked distractor effects on position judgments. Each subject provided position judgments on 30 masked trials at the exposure duration that was to be used for the subsequent masked priming trials. As previously described, the procedures obliged performance to be at least slightly below chance accuracy on these trials. In order to obtain a test for detectionless processing, the position judgments were analyzed to determine what percentage of them agreed with the task-irrelevant semantic content of the word (LEFT or RIGHT) that was presented on each trial. By chance, $50 \%$ of responses should have been congruent with the irrelevant word. However, $57.8 \%$ (SEM $=1.33 \%)$ of responses were congruent, significantly more than chance $[F(1,19)=34.26, p<.001]$. This finding provides converging evidence for the processing of undetected word stimuli.

\section{Discussion}

In Experiment 1 we sought to test for detectionless processing of word meaning, and to do so using a stringent criterion for establishing that masked stimuli could not be detected (using the position-judgment task) and two novel measures of semantic processing (priming of evaluative decisions and influence of irrelevant content of masked words on position judgments). Both tests produced evidence for semantic processing. The findings therefore tend to support the controversial conclusions of the previous studies by Marcel (1983), Fowler et al. (1981), and Balota (1983).

\section{Attempted Replications of Experiment 1}

Puzzlingly, three attempted replications of Experiment 1 yielded inconsistent findings: either findings like

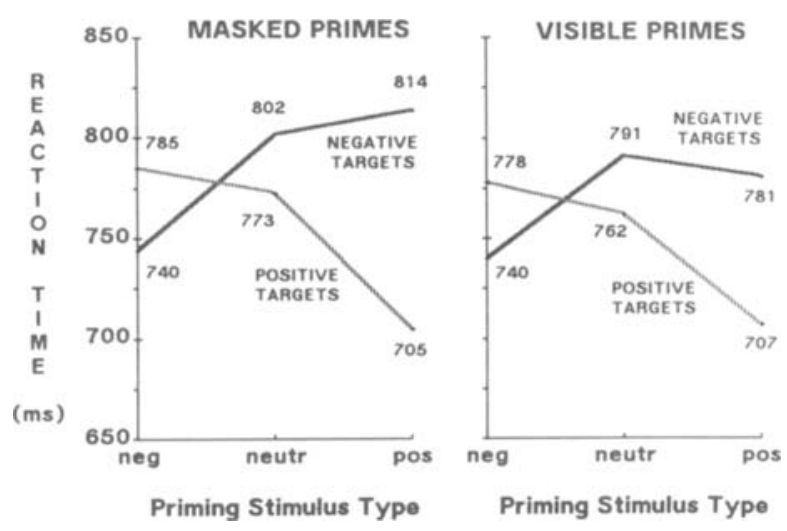

Figure 3. Mean latencies for evaluative decisions, Experiment 1 $(n=20)$. 
those of Experiment 1 were obtained, or no priming effects at all were obtained-even with presumably visible (unmasked) presentation of the priming stimuli. A check of the illumination levels of the tachistoscope indicated a drop from the levels measured during Experiment 1, probably due to bulb aging. However, other apparatus factors may have been involved, due to the tachistoscope's having been moved from one laboratory to another after Experiment 1. After the difficulty in replicating Experiment 1 , it was observed that the 10 -msec wordpresentation durations, which previously had permitted legibility of words presented to the nondominant eye, no longer sufficed. This observation prompted a substantial revision of the stimulus presentation procedures for the following experiments. (Additionally, a test of the speculation that illumination duration of primes was important to the masked priming effect was incorporated into Experiment 3.)

\section{EXPERIMENT 2}

In Experiment 1, as well as in the previous studies by Marcel (1983), Fowler et al. (1981), and Balota (1983), the masking procedure used was dichoptic backward masking-presentation of the priming stimulus to the nondominant eye, followed within 10 to $30 \mathrm{msec}$ by presentation of a pattern mask to the dominant eye. It seemed possible that the masking effect of this procedure might be dependent more on binocular rivalry (with the dominant eye taking precedence in perception of approximately simultaneous stimuli) than on "erasure" (with the second stimulus interrupting processing of the first; see Walker, 1978; Wolfe, 1986). This reasoning prompted use, in Experiment 2, of a procedure in which the prime and mask were presented simultaneously-the prime to the nondominant eye and the mask to the dominant eyeallowing longer duration of undetectable primes. Two other modifications of procedure simplified the conduct of the experiment. First, the initial period of dark adaptation used in Experiment 1 was eliminated because it was unnecessary (because the durations of illuminated stimuli were enough to undo dark adaptation). Second, because the prime and mask were now to be presented simultaneously, the lengthy procedure previously used to establish individual prime-target onset intervals was not needed.

\section{Method}

Subjects. The subjects were 12 volunteers, male and female, who participated in partial fulfillment of a requirement of their introductory psychology course. Data for an additional 4 volunteers were not included in analyses, 2 because of apparatus failures and 2 because their performance on the position-discrimination tasks (described below), which were interspersed through the experiment, indicated that the priming stimuli were at least partially visible to them.

Apparatus and Stimuli. The apparatus (with tachistoscope bulbs replaced) and, with one exception, the stimuli used for all phases of the experiment were the same as those used in Experiment 1. The exception was that blank stimuli were used in place of evalua- tively neutral priming words. This change was made because of concern that the evaluatively neutral words used previously might not be similar to the evaluatively polarized words in all respects other than level of evaluation. (There are, of course, reasons for similar concern that blanks might not provide a proper neutral baseline. The change is best viewed as an alternative approach to establishing baselines.)

Presentation-duration determination. After the subject's dominant eye was determined and the tachistoscope's eyepiece polarizing filters were placed accordingly, each subject was asked to read a series of single (evaluatively neutral) words presented to the nondominant eye for brief durations, while the dominant eye was exposed to a dark field. The tachistoscope was initially set to present these words for a duration of $500 \mathrm{msec}$, and this duration was decreased through values of $200,100,80,60,50,40,30,25$, 20 , and $15 \mathrm{msec}$, after every third word, until the subject first missed a word. The duration was then kept at the same value for six more words. If the subject missed more than one of these six, the duration assigned to the subject was the preceding one (at which three out of three had been read correctly). In effect, then, the selected duration was one at which the subject read at least seven of nine words correctly. The selected presentation durations ranged, across the group of 12 subjects, from 30 to $100 \mathrm{msec}$ (median $=60 \mathrm{msec}$; $\mathrm{cf}$. the 10-msec duration used in Experiment 1, and see the discussion of attempted replications of Experiment 1).

Position-discrimination test. The tachistoscope was used to present test words to the nondominant eye and the pattern mask simultaneously to the dominant eye (see Figure 4 ). These were presented for the duration previously determined to be one at which the subject could read single words that were presented only to the nondominant eye. The subjects were told that a word would be presented either just left or just right of the fixation point on each trial. They were asked to indicate the right or left position of the word on each trial by pressing the corresponding button. Twentyfour test trials, consisting of a random series of the words LEFT and RIGHT presented either left or right of center, were conducted in this fashion. This test was repeated twice more during the experiment (each time using the 24 test stimuli in a different order), once after three of six blocks of priming test trials, and again at the end of the last block of priming test trials.

Masked and visible priming tests. There were six blocks of 40 trials of the evaluative decision task, three conducted with masked primes and three with visible primes. Each block started with four warmup trials, for which data were not analyzed. The following 36 trials included 6 each of the six combinations of three types of primes (positive, negative, and blank) with two types of targets (positive and negative). ${ }^{5}$ Six different blocks of stimuli were prepared. These were administered in an order that was different for

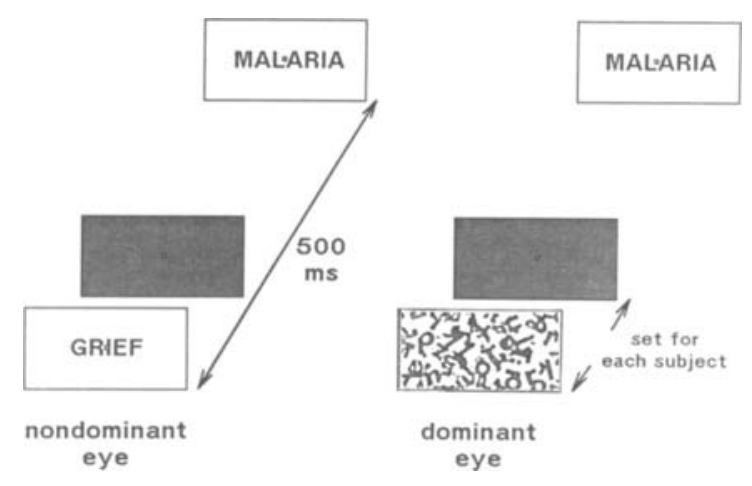

Figure 4. Procedure for priming by simultaneous dichoptically masked words, Experiment 2. 
each subject and was counterbalanced, over the set of 12 subjects, for order of stimulus blocks, the assignment of stimulus blocks to treatments, and the order of masked and visible priming treatments. Detection tests, as previously described, were conducted after the third and sixth block of trials.

\section{Results}

Position-discrimination accuracy. Two subjects performed well enough on the position-discrimination tests to establish that the simultaneous dichoptic masking procedure did not make priming stimuli undetectable for them. (These 2 were replaced by other subjects in the counterbalancing design of the experiment.) For the 12 subjects whose data were retained, performance on the 72 positiondiscrimination test trials distributed throughout the session averaged $51.4 \%$ correct (chance $=50 \%$ ), ranging from a minimum of $45.8 \%$ ( 33 of 72 ) to a maximum of $63.9 \%(46$ of 72$){ }^{6}$

Evaluative decision task. Errors occurred on $6.8 \%$ (SD $=3.6 \%$ ) of the evaluative-decision trials, and were slightly (but not significantly) more frequent in the visible priming $(8.2 \%)$ than in the masked priming $(5.3 \%)$ condition. Error rates were not significantly affected by prime-target congruence in either condition. The analyses reported are based on correct-response trials only; however, analyses based on all trials, including errors, were also computed, and showed the same pattern of findings. As in Experiment 1, a trimming procedure was used to eliminate latencies outside the range of $400-1,600 \mathrm{msec}$ (3.1\% of all responses). Most of these outliers were long latencies caused by occasional tachistoscope card-changer malfunctions.

Priming effects. Figure 5 presents the evaluativedecision latency data for both masked and visible priming conditions. The most important aspect of the results is their demonstration of priming effects of stimuli that were presumed to have been rendered undetectable by simultaneous dichoptic masking. In the masked priming condition, the overall test of priming effects, which examined the difference between mean latencies in congruent and incongruent priming conditions $(799-765=$

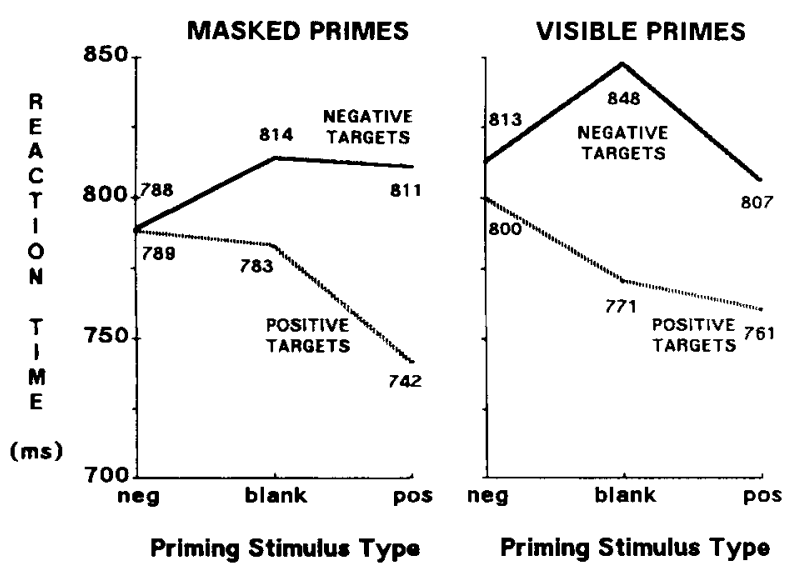

Figure 5. Mean latencies for evaluative decisions, Experiment 2 $(n=12)$.
$34 \mathrm{msec}, S E M=8.6 \mathrm{msec}$ ), yielded a highly significant effect $[F(1,11)=15.28, p<.001]$. Finer level analyses indicated that only the facilitative priming effect of positive primes on positive targets was individually statistically significant in the masked priming condition. In the visible priming condition, the difference between congruent and incongruent priming conditions (803 - 787 $=16 \mathrm{msec}, S E M=10.0 \mathrm{msec}$ ) was not statistically significant $[F(1,11)=2.58$, n.s. $]$. Tests at the finer levels of analysis (for facilitation by congruent primes and interference by incongruent ones) also indicated no statistically significant effects.

Masked distractor effects on position discrimination. Each subject provided responses on 72 positiondiscrimination test trials, presented in three blocks of 24 trials each at the beginning, middle, and end of the experimental session. The subjects' responses on an average of $52.8 \%(S E M=1.85 \%)$ of these trials agreed with the irrelevant, and presumably not visible, distracting word. This agreement with semantic content was greater than chance level, but not significantly so $[F(1,11)=$ $2.49, p<.15]$.

\section{Discussion}

The major result of Experiment 2 was the demonstration of facilitative priming of evaluative decisions by words that were masked to prevent their detection (by the criterion of chance accuracy at position discrimination). This confirmed the finding of Experiment 1, while replacing Experiment 1's backward dichoptic pattern-masking procedure with a simultaneous dichoptic pattern-masking procedure that permitted increased presentation duration of the masked word stimuli. The second test, for the effect of nondetectable distracting word stimuli on position judgments, was consistent in direction with a detectionless processing effect, but was not statistically significant. Unexpectedly, Experiment 2 did not yield a statistically significant effect of visible priming. This result was nevertheless consistent with the results of Experiment 1 (as well as those of Experiment 3, below) in that 95\% confidence intervals (approximately $\pm 2 S E M$ ) on the obtained priming effects of the three experiments overlap.

\section{EXPERIMENT 3}

The simultaneous dichoptic pattern-masking procedure of Experiment 2 was developed to permit a lengthened presentation of masked stimuli. The apparent success of this procedure reinforced the authors' suspicion that the inconsistent results of initial attempts to replicate Experiment 1 might have been due to short priming-stimulus exposure durations, coupled with the low illumination levels available in the Gerbrands tachistoscope. Experiment 3 was conducted primarily to assure the replicability of the masked priming effect that was obtained with Experiment 2's simultaneous masking procedure. A secondary aim was to test two hypotheses about conditions on which detectionless processing of evaluative meaning might de- 
pend. First, it was expected that priming by masked undetectable stimuli might depend on the exposure duration of the masked stimuli. Accordingly, in Experiment 3 two exposure durations were used for masked priming stimuli. Second, it was hypothesized that the priming effects of Experiments 1 and 2 might have depended on the great overlearning of evaluative meaning that occurs for words that occur frequently in the language. Therefore, Experiment 3's design used priming words that fell into two distinct ranges of word frequency.

\section{Method}

Subjects. The subjects were 12 volunteers from the same population used for Experiments 1 and 2. Data for an additional 4 volunteers were not included in analyses, 3 because they were not easily able to read words presented for $80 \mathrm{msec}$ to their nondominant eyes, and $I$ because performance on position-discrimination tests (see below) indicated that masked priming stimuli presented for $80 \mathrm{msec}$ were at least partially visible.

Apparatus and Stimuli. The apparatus was the same as that used in Experiments 1 and 2, and the target stimuli for the evaluative decision task were selected as in the previous experiments. However, the design was modified in regard to priming stimuli. No neutral primes were used. (This prevented collection of further data on the occurrence of facilitative vs. interference priming effects; however, use of neutral primes in combination with the other designindependent variables would have expanded the within-subjects design beyond what could be accomplished in a single experimental session.) The 12 words used as priming stimuli were in four subcategories: (1) high frequency, positive (joy, honest, vacation); (2) moderate frequency, positive (hug, terrific, affectionate); (3) high frequency, negative (hate, prison, terrible); and (4) moderate frequency, negative (ulcer, sickness, torture). Word frequencies were obtained from the Kucera and Francis (1967) norms. High-frequency words had frequencies in the range of 40-47 per million, whereas moderate-frequency words had frequencies in the range of 3-6 per million. As before, the positive and negative priming stimuli were chosen from the positive and negative extremes, respectively, of the Bellezza et al. (1986) norms for judged pleasantness.

Procedure. After determination of eye dominance, the subjects were tested for their ability to read evaluatively neutral single words presented for brief durations to the nondominant eye. The presentation duration was lowered gradually from 500 to $80 \mathrm{msec}$. Only data from subjects who could read at least six of eight words presented for $80 \mathrm{msec}$ to the nondominant eye were retained for use in the major hypothesis tests. (As previously noted, data for 3 subjects were not included in analyses because they did not meet this criterion.) Next, subjects received the first of three 24-trial tests for position discrimination of masked stimuli. These tests and their early-, middle-, and late-session placements were the same as in Experiment 2, except that the duration of positioned test words and simultaneous masks was fixed at $80 \mathrm{msec}$ for all subjects. (One subject was replaced because of ability to see the positions of these test words.)

For priming tests, data were obtained for three treatments: 40msec masked (i.e., prime and mask presented simultaneously for $40 \mathrm{msec}$ ), 80 -msec masked (prime and mask presented simultaneously for $80 \mathrm{msec}$ ), and $80-\mathrm{msec}$ visible (prime presented alone, to the nondominant eye, for $80 \mathrm{msec}$ ). The two masked priming durations were, respectively, $20 \mathrm{msec}$ shorter and $20 \mathrm{msec}$ longer than the median duration used for the subjects in Experiment 2. Each of the three priming treatments appeared in one block of 40 trials between the first and second position-discrimination tests, and in another block of $\mathbf{4 0}$ trials between the second and third tests.
Each subject received a different order of materials that counterbalanced order of presentation of six different blocks of stimuli, assignment of stimulus blocks to priming treatments, and order of priming treatments. As in Experiments 1 and 2, the first four trials in each block of priming trials served as unanalyzed practice trials.

\section{Results}

Position-discrimination accuracy. These tests were conducted in three blocks of 24 trials, using simultaneous, dichoptic 80-msec presentations of mask and positioned test words for all subjects. For the 12 subjects whose data were retained, average correct position-discrimination performance was $52.0 \%$, ranging from a low of $41.7 \%$ (30 of 72) to a high of $58.3 \%$ (42 of 72).

Evaluative decision task. Errors on the evaluative decision task occurred on an average of $9.9 \%(S D=9.2 \%)$ of trials, and were slightly (not significantly) greater in the 80 -msec visible priming condition $(10.8 \%)$ and 40 msec masked priming condition $(11.7 \%$ ) than in the 80 msec masked condition (7.3\%). Error rates were not significantly affected by prime-target congruence or incongruence in any of the three priming conditions. The analyses reported were based on correct-response trials. (The same pattern of findings was obtained from analyses that included error trials.) Latency distributions were trimmed as in Experiments 1 and 2 by eliminating latencies shorter than $400 \mathrm{msec}$ and longer than $1,600 \mathrm{msec}$ (eliminating $3.3 \%$ of correct-response trials).

Priming effects. Figure 6 presents the evaluativedecision latency data for the three priming conditions. For each condition, a measure of the priming effect was obtained by subtracting the mean decision latency for congruent prime-target combinations (positive-positive and negative-negative) from the mean latency for incongruent combinations (positive-negative and negative-positive). This priming effect was significantly greater than zero for the 80 -msec masked condition $[733-694=39 \mathrm{msec}$, $S E M=16.2 \mathrm{msec} ; F(1,11)=5.89, p<.05]$ and for the $80-\mathrm{msec}$ visible condition $[726-682=44 \mathrm{msec}$, $S E M=11.4 \mathrm{msec} ; F(1,11)=8.80, p<.05]$, but not for the 40-msec masked condition $[670-654=16 \mathrm{msec}$, $S E M=12.6 \mathrm{msec} ; F(1,11)=2.02, p>.15]$. The prim-

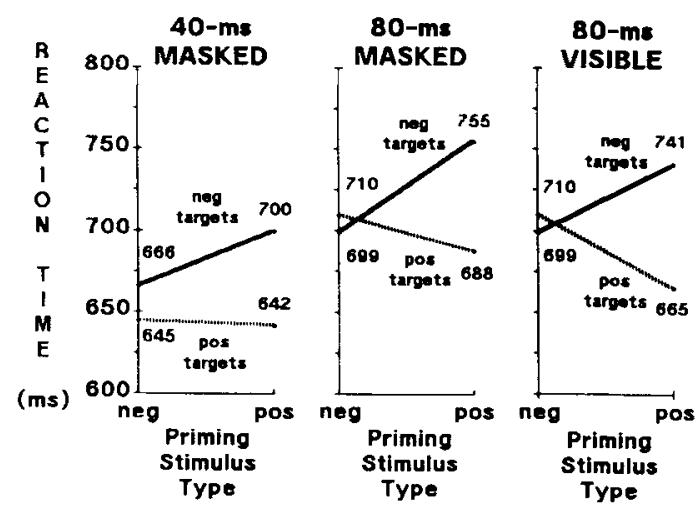

Figure 6. Mean latencies for evaluative decisions, Experiment 3 $(n=12)$. 
ing effect in the 80-msec masked condition ( $39 \mathrm{msec}$ ) was greater than that in the 40 -msec masked condition (16 msec); however, this difference was not statistically significant $(F<1)$.

Effect of word frequency of priming words. On the basis of the assumption that priming might be a consequence of highly overlearned word meanings, it was expected that priming should be greater for priming words with higher frequencies. On the contrary, however, priming effects were slightly (not significantly) greater for moderate-frequency than for high-frequency priming words. Over all three priming conditions, the mean priming effect averaged $31 \mathrm{msec}$ for high-frequency priming words and $35 \mathrm{msec}$ for moderate-frequency priming words.

Masked distractor effects on position judgments. Each subject provided data on 72 position-judgment trials, distributed across the experimental session in three blocks. An average of $53.8 \%$ ( $S E M=1.67 \%$ ) of these responses were congruent with the irrelevant, undetectable word stimulus (LEFT or RIGHT). This was significantly greater than chance $[F(1,11)=5.04, p<.05]$.

\section{Discussion}

The detectionless processing effects found in Experiment 3 are important to the present research in three ways. First, they establish that the priming effect obtained with Experiment 2's simultaneous dichoptic pattern-masking procedure is replicable. Second, the tendency (even though nonsignificant) for priming effects to be larger with longer duration of masked primes is consistent with the authors' speculation that brief illumination durations may have been responsible for the previous difficulty in replicating Experiment 1 . Third, the influence of masked distracting words on position judgments replicated Experiment 1's similar significant finding. Over the three experiments, the detectionless processing effect on position judgments was highly significant, with an average of $55.3 \%$ (SEM $=0.90 \%$ ) of responses agreeing with the masked distracting word $[F(1,43)=30.98, p<.001]$.

\section{GENERAL DISCUSSION}

Previous research on detectionless processing has been regarded as empirically ambiguous. Accordingly, the main focus of the present research was on determining whether there are any conditions under which detectionless processing of word meaning can be demonstrated reliably. Two procedurally independent tasks, judging evaluative meanings of masked words and judging the location of masked words, reliably demonstrated processing of word meaning under conditions of dichoptic pattern masking.

Masked priming of evaluative decisions was statistically significant in all three experiments; responses to evaluatively polarized target words preceded by evaluatively congruent masked primes were faster than responses to targets preceded by evaluatively incongruent masked primes. Additionally, in Experiments 1 and 3 (and for the series of experiments overall), judgments of the left or right location of masked words (observed on threshold testing trials) agreed at a significantly above-chance rate with the semantic content of masked words that could not be located with above-chance accuracy. These two replicable findings converge with the findings of masked priming effects in previous studies that used dichoptic pattern masking in conjunction with lexical decision tasks (Balota, 1983; Fowler et al., 1981; Marcel, 1983).

The demonstration of semantic processing of masked words with the present position-discrimination task is of special interest because (1) position judgments were influenced on the very same series of trials on which subjects were shown to be performing at chance in their position judgments, and (2) the apparently invisible words influenced the actual choice of response. In this respect, Klatzky $(1984$, p. 52) noted, in discussing effects of stimuli presented outside of awareness, that effects on speed of cognitive processes (such as masked priming effects) do not imply effects on choice behavior, and effects on choice behavior had never been demonstrated (i.e., prior to the present data).

\section{Present Findings in Light of Methodological \\ Criticisms of Previous Studies}

The present experiments were designed to be responsive to two criticisms of the previous studies by Marcel (1983), Fowler et al. (1981), and Balota (1983). Those two criticisms focused on illumination patterns and detectability criteria. It now seems possible to dismiss entirely the illumination-pattern basis for criticism, and to conclude that the detectability-criterion problem is much diminished by the present findings.

Illumination patterns. Purcell et al. (1983) demonstrated that relatively low contextual illumination levels used during threshold-setting procedures in a study by Carr, McCauley, Sperber, and Parmelee (1982) selected presumed threshold conditions that plausibly permitted word primes to be visible under the higher contextual illumination used for later masked priming tests. Holender (1986) argued that this criticism applied also to the studies by Marcel (1983), Fowler et al. (1981), and Balota (1983). In the present experiments, however, any possibility of an illumination-pattern artifact was eliminated by using identical patterns of illumination for thresholdtesting (position-discrimination) trials and masked priming trials. Balota (1983) had similarly avoided illuminationpattern problems. Accordingly, the illumination-pattern criticism can confidently be dismissed, at least in regard to four demonstrations (Balota's and the present three) of detectionless processing of word meaning.

Detectability criteria. Cheesman and Merikle (1985) suggested that the threshold-setting procedures used in previous studies may have selected exposure durations at which, subjects' reports of not seeing test stimuli notwithstanding, some stimulus detection could occur. Perhaps, that is, subjects either felt more comfortable saying "ab- 
sent" than "present" when they could not see test stimuli clearly or confidently, or adopted a strategy of deliberately randomizing present and absent judgments when the stimuli were not clearly detectable. Either of these responding strategies could give the appearance of stimulus nondetectability $\left(d^{\prime} \cong 0\right)$ under conditions in which partial detection might have been apparent with other response strategies. The present experiments dealt with this detectability-criterion problem in two ways. First, Experiment 1 required that each subject's positiondiscrimination performance fall below $50 \%$ accuracy, in selecting stimulus presentation conditions to be used for masked priming tests. (This does not mean that the conditions of Experiment 1 sought to establish the nonsensical condition of $d^{\prime}<0$. Rather, the intent was to reduce the possibility of a true $d^{\prime}>0$ for subjects who were retained in the experiment.) Second, in Experiments 2 and 3 , position-discrimination tests were administered before, during, and after masked priming tests; any subject whose performance was clearly above chance for some subset or all of the 72 test trials was excluded from tests of masked priming effects.

The present procedures were capable of detecting with a probability of about 0.50 (at a one-tailed .05 significance level) a subject who had a true $d^{\prime}$ of 0.50 for the 72-trial position-discrimination test. (A $d^{\prime}$ of 0.50 corresponds to an expected position-discrimination performance of about $60 \%$ correct.) Considered in this fashion, the present procedures do not appear to offer much assurance that each subject was unable to discriminate stimulus position. However, it is more appropriate to consider the present procedures' sensitivity to mean levels of $d^{\prime}$ for the group of subjects. The mean performance of a group of 12 subjects (the $N$ for Experiments 2 and 3) can be used to detect a population mean $d^{\prime}$ of 0.20 (corresponding to $54 \%$ correct performance) with a respectable probability of 0.80 . A population mean $d^{\prime}$ of 0.15 (53\% correct) can be detected with a probability of 0.80 by the average of 24 subjects (the combined $N$ s for Experiments 2 and 3 ).

Two subjects in Experiment 2 and 1 in Experiment 3 were rejected because of their accurate positiondiscrimination performance $(78 \%, 82 \%$, and $71 \%$ accuracy, respectively). The average position-discrimination accuracy for 27 subjects (the 24 for whom results were reported in addition to the 3 rejected) was $54.3 \%$, which corresponds to a $d^{\prime}$ of 0.22 (maximum likelihood estimate). The 3 rejected subjects (average accuracy = $76.9 \%$ ) were clearly outliers relative to the distribution of accuracy scores for the 24 retained subjects (average accuracy $=51.5 \%, S D=5.0 \%$ ). Elimination of the 3 outliers provides a basis for confidence that mean $d^{\prime}$ for the remaining 24 was considerably lower than 0.22 , perhaps close to zero (depending on one's assumption about the population variability of $d^{\prime}$ ). Of course, no threshold test can provide absolute confidence that $d^{\prime}$ is zero. The present procedures warrant the conclusion that the 24-subject mean $d^{\prime}$ for position discrimination in Ex- periments 2 and 3 was, at most, very low, plausibly less than 0.1 .

\section{Further Consideration of Detectability Criteria}

In any experiment that uses masked priming procedures, the subject's task on detection test trials is necessarily different from that on masked-priming test trials. In the present experiments, for example, detection trials used a right/left position-discrimination task (which had been adopted in the face of Merikle's, 1982, criticisms of the presence/absence judgment task that was used in some previous experiments). Possibly, stimulus presentation conditions that produced chance performance on the present position-discrimination task could have produced above-chance accuracy on some other discrimination task. For example, the test stimuli might have been colored red and green, and subjects might have been asked to make a color discrimination; or the stimuli might have been 00000 and XXXXX, with subjects being asked to discriminate curvature or linearity.

There seems to be only one satisfactory response to criticisms of detectionless processing research that point to differences between detection test and priming test tasks-and that is to recognize that these criticisms are valid. In the case of the present experiments, for example, it cannot be asserted that subjects would have failed to discriminate features other than position. ${ }^{8}$ In the case of previous studies that have used presence/absence judgments on threshold tests, it cannot be asserted that the presentation conditions were such that subjects would have failed to discriminate, say, color, curvature, or stimulus position. More generally, no amount of negative evidence on a diverse series of discrimination tests can justify the conclusion that some other, not yet tested, feature is undetectable. Accordingly, appropriate caution requires that the present conclusion of detectionless processing for the evaluative decision task be qualified by an understanding that "detectionless" was operationally defined as chance performance on position discrimination of word stimuli. The conclusion is nevertheless a strong one, because it has been demonstrated (see Appendix) that exposure durations that render word position undetectable also render word presence versus absence undetectable, and are more stringent (i.e., briefer or dimmer) than those required for identification of graphemic (letter or word) features.

\section{Theoretical Interpretation}

Establishment of the empirical conditions on which a finding depends necessarily has theoretical implications. Any theory that predicts the conditions receives a strong boost, theories that can offer an unstrained account of the condition dependency receive a mild boost, and theories that cannot readily explain the observed limiting conditions are at least mildly discredited. Semantic processing of masked words has now been demonstrated repeatedly with dichoptic pattern-masking procedures, in which word and mask are presented to different eyes either simulta- 
neously (as in the present Experiments 2 and 3) or with word preceding mask by intervals of about $10-30 \mathrm{msec}$ (as in the present Experiment 1 and in the previous studies by Marcel, 1983, Fowler et al., 1981, and Balota, 1983). By contrast, semantic processing of masked words has not been demonstrated replicably with monocular or binocular masking, in which word and (pattern or energy) mask are presented to the same eye(s). (Only Marcel, 1983, explicitly contrasted dichoptic pattern-masking and monocular energy-masking procedures in the same experiment; he obtained significant masked priming of lexical decisions only with the dichoptic masking procedure.)

One interpretation of the difference between same-eye (monocular or binocular) and different-eye (dichoptic) masking procedures is that only the latter permits access of masked visual stimuli to central processing (Turvey, 1973). However, because this interpretation of dichoptic pattern masking is itself controversial (see Bridgeman, 1986; Kahneman, 1968), additional findings of limiting conditions on semantic processing of masked words will be needed to achieve greater progress in theoretical interpretation. The best leads provided from the present research come from the relative ease of obtaining the effect with the simultaneous dichoptic masking procedure of Experiments 2 and 3, in comparison with the backward dichoptic masking procedure of Experiment 1 (which was inconsistently replicated). A major difference between these two procedures was that the simultaneous masking procedure permitted longer presentation durations of undetectable stimuli. Additional evidence suggesting that priming effects depend on exposure duration comes from the finding in Experiment 3 of a larger (but nonsignificantly so) masked priming effect for 80 -msec than for 40 -msec exposure durations. Should further research confirm the dependency of masked priming effects on exposure duration, theories that appeal to time-dependent processes (e.g., spreading activation) will be favored.

Studies using variations of priming word stimuli may prompt discoveries of further constraints on theory. In the present research, there was some evidence that facilitation (congruent priming) was more effective than interference (incongruent priming) for masked primes. Another stimulus variation used in the present research, the variation of word frequency of priming stimuli in Experiment 3 , did not affect magnitude of priming. However, it remains possible that more extreme variations of priming-word frequency would be effective.

\section{FINAL COMMENT}

The authors embarked on this series of experiments as skeptics who were prepared to seek publication of null findings if null results could be consistently obtained (cf. Greenwald, 1975), but, at the same time, were impressed that three independent previous studies had produced mutually supportive evidence for possibly detectionless processing of word meaning (Balota, 1983; Fowler et al.,
1981; Marcel, 1983). The present experiments, taken in conjunction with those earlier investigations, provide strong converging evidence that the procedure of dichoptic pattern masking effectively yields evidence for detectionless processing of word meaning, and may do so more reliably when used with simultaneous masking than with backward masking.

\section{REFERENCES}

ANDERson, J. R. (1982). Acquisition of cognitive skill. Psychological Review, 89, 369-406.

Balota, D. A. (1983). Automatic semantic activation and episodic memory encoding. Journal of Verbal Learning \& Verbal Behavior, 22, 88-104.

Bellezza, F. S., Greenwald, A. G., \& Banai, M. R. (1986). Words high and low in pleasantness as rated by male and female college students. Behavior Research Methods, Instruments, \& Computers, 18, 299-303.

Bridgeman, B. (1986). Theories of visual masking. Behavioral \& Brain Sciences, 9, 25-26.

Carr, T. H., McCauley, C., Sperber, R. D., \& Parmelee, C. M. (1982). Words, pictures, and priming: On semantic activation, conscious identification, and autonaticity of information processing. Journal of Experimental Psychology: Human Perception \& Performance, 8, 757-777.

Cheesman, J., \& Merikle, P. M. (1985). Word recognition and consciousness. In D. Besner, T. G. Waller, \& G. E. MacKinnon (Eds.), Reading research: Advances in theory and practice (Vol. 5, pp. 311352). New York: Academic Press.

Cheesman, J., \& Merikle, P. M. (1986). Distinguishing conscious from unconscious perceptual processes. Canadian Joumal of Psychology, 40, 343-367.

Duensing, S., \& Miller, B. (1979). The Cheshire Cat effect. Perception, 8, 269-273.

ERdelyi, M. H. (1974). A new look at the new look: Perceptual defense and vigilance. Psychological Review, 81, 1-25.

ERIKSEN, C. (1958). Unconscious processes. In M. R. Jones (Ed.), Nebraska Symposium on Motivation: 1958. Lincoln: University of Nebraska Press.

Fazio, R. H., Sanbonmatsu, D. M., Powell, M. C., \& Kardes, F. R. (1986). On the automatic activation of artitudes. Journal of Personality \& Social Psychology, 50, 229-238.

Fisher, R. A., \& CRAIK, F. I. M. (1977). Interaction between encoding and retrieval operations in cued recall. Journal of Experimental Psychology: Human Learning \& Memory, 3, 701-711.

Fowler, C. A., Wolford, G., Slade, R., \& Tassinary, L. (1981). Lexical access with and without awareness. Journal of Experimental Psychology: General, 110, 341-362.

GreEnwaLD, A. G. (1975). Consequences of prejudice against the null hypothesis. Psychological Bulletin, 82, 1-20.

HOLENDER, D. (1986). Semantic activation without conscious identification in dichotic listening, parafoveal vision, and visual masking: A survey and appraisal. Behavioral \& Brain Sciences, 9, 1-66.

KaHNEMAN, D. (1968). Method, findings, and theory in sudies of visual masking. Psychological Bulletin, 70, 404-425.

KLATZKY, R. L. (1984). Memory and awareness: An informationprocessing perspective. New York: Freeman.

KuČERA, H., FRANCIS, W. N. (1967). Computational analysis of present-day American English. Providence, RI: Brown University Press.

MARCEL, A. J. (1983). Conscious and unconscious perception: Experiments on visual masking and word recognition. Cognitive Psychology, 15, 197-237.

MERIKLE, P. M. (1982). Unconscious processing revisited. Perception \& Psychophysics, 31, 298-301.

Meyer, D. E., \& Schyaneveldt, R. W. (1971). Facilitation in recog- 
nizing pairs of words: Evidence of a dependence between retrieval operations. Journal of Experimental Psychology, 90, 227-234.

Moore, T. (1982). Subliminal advertising: What you see is what you get. Journal of Marketing, 46, 38-47.

Moray, N. (1970). Attention: Selective processes in vision and hear ing. New York: Academic Press.

Osgood, C. E., Suci, G., \& Tannenbaum, P. H. (1958). The measurement of meaning. Urbana, IL: University of Illinois Press.

Purcell, D. G., Stewart, A. L., Stanovich, K. E. (1983). Another look at semantic priming without awareness. Perception \& Psychophysics, 34, 65-71.

Rock, I., GuTMan, D. (1981). The effect of inattention on form perception. Joumal of Experimental Psychology: Human Perception \& Performance, 7, 275-285.

TURVEY, M. T. (1973). On peripheral and central processes in vision: Inferences from an information-processing analysis of masking with patterned stimuli. Psychological Review, 80, 1-52.

WALKER, P. (1978). Binocular rivalry: Central or peripheral selective processes? Psychological Bulletin, 85, 376-389.

WOLFE, J. M. (1986). Stereopsis and binocular rivalry. Psychological Review, 93, 269-282.

\section{NOTES}

1. In this article, references to detectability and thresholds do not imply the conception of an absolute or fixed energy level at which a stimulus is reliably detectable (and below which it is not). Rather, the prevailing statistical sense of threshold is understood, according to which an undetectable stimulus is one for which the signal detection theory measure of detectability, $d^{\prime}$, is indistinguishable from zero.

2. Although the position-discrimination task was introduced in order to avoid criticisms of the presence-absence judgment task, two reviewers pointed out that the task of discriminating stimulus left/right position might be more difficult than that of judging stimulus presence/absence. If so, the criterion of chance performance on the position-discrimination task might select presentation conditions that would permit presence/ absence or (importantly) other features to be detected. Because of the potential validity of this criticism, additional data were collected to com pare the ease of detecting stimulus right/left position, stimulus presence/absence, and (while we were at it) letter and word features These data are reported in the Appendix. The additional data indicate that the task of detecting right/left position was approximately equivalent in difficulty to that of judging word presence/absence, and that both of these tasks were considerably easier (not more difficult) than detecting letter or word features. Therefore, stimulus presentation conditions that reduce position discrimination or presence/absence judgments to chance levels should effectively exclude the detection of graphemic features.

3. This procedure was conservative in three senses. First, the requirement of more than $50 \%$ errors ensured that subjects could not meet the criterion simply by giving a constant left or right response on all trials. (However, no subject used such a constant-response strategy.) Second, the high error criterion reduced the likelihood that subjects who perceived stimuli on some trials, but guessed unluckily on other trials, could achieve the criterion. Third, the procedure necessarily selected somewhat shorter exposure durations than would have been selected by a criterion of $50 \%$ accuracy. This conservatism was prompted by published comments that previous researchers' threshold-determination procedures may have selected exposure conditions that permitted some detection.

4. The manner in which the data were recorded on diskette did not permit determination of which latencies were for correct responses and which for errors. This oversight was corrected for the subsequent experiments, which also indicated that patterns of results were identical for errors-included and errors-excluded analyses.

5. It was later discovered that not all of the blocks of 36 trials were fully balanced, as intended, for sequences of pairs of trial types (cf. procedure of Experiment 1). This procedural error turned out not to have any impact on results, as indicated by the lack of any significant or near-significant effects involving prior trial type in a preliminary analysis.

6. Performance accuracy of $63.9 \%$ correct exceeded a one-tailed (but not a two-tailed) .05-level significance criterion for positiondiscrimination accuracy. It can therefore be argued that this subject should have been omitted from analyses. Accordingly, all significance tests reported below were recomputed without this subject's data. No significance test outcomes were altered by omitting this subject.

7. An extreme form of the argument about detectability criteria is that the occurrence of masked priming in the present experiments itself constitutes evidence that information from the priming stimuli was detected. However, such a broad use of the verb detect is problematic. In particular, a huge body of existing psychophysical research must be deemed irrelevant to the study of detection if verbal report of presence/absence is judged inadequate as an operational definition of detection. Furthermore, the very enterprise of seeking evidence for processing of meaning in the absence of detection becomes pointless.

8. Indeed, informal tests with a few subjects indicated that it was possible to discriminate positions of color patches and to identify their colors (but not to see or identify black letters that were superimposed on detectable color patches) under the masking conditions that made word positions undetectable in the present research. But soe the Appendix for further evidence that letter and word features were almost certainly undetectable in the present experiments.

\section{APPENDIX \\ Comparison of Position Discrimination and Presence/Absence Judgment as Measures of Detection}

After completion of the three experiments reported in the main text, data were collected to evaluate the position-discrimination task that was used to assess detection thresholds in the present study. In particular, the position-discrimination task was compared with that of judging word presence or absence (the task used in most previous masked priming research), as well as with the tasks of discriminating letters and words. These additional data were collected out of concern that, if position is more difficult to detect than other visible features (e.g., presence/ absence or graphemic features), then thresholds set on the basis of position discrimination might permit detection of such other features.

The three supplementary data collections described here employed stimulus presentation conditions that would permit low to moderate levels of accurate detection, in order to permit comparison of the difficulties of the several judgment tasks that were examined. Data Set 1 was obtained using the same apparatus used in the three main experiments. As a consequence of the authors' move from Ohio State University to the University of Washington, Data Sets 2 and 3 were obtained with different equipment, which is described below.

\section{Data Set 1}

Sixteen subjects provided data for $\mathbf{4 8}$ trials on each of two tasks, position discrimination and graphemic word discrimination. All stimuli were dichoptically masked (using the simultaneous masking procedure), and were $60 \mathrm{msec}$ in duration. The position-discrimination task was the same as that used in Experiments 1,2, and 3 (i.e., the subject was to judge whether the word RIGHT or LEFT was presented to the right or left of center on each trial). Stimuli for the graphemic word discrimination task were words from a list of synonym pairs prepared by Fisher and Craik (1977). Immediately after masked 
presentation of one word from a synonym pair, the subject was shown both items of the pair and was asked to choose the previously presented word. (Synonym stimuli were chosen in light of the possibility that masked presentations might transmit semantic features independently of graphemic ones.) Subjects performed somewhat, but not significantly, better on position discrimination than on graphemic word discrimination [mean $d^{\prime} \mathrm{s}=0.70$ and 0.48 , respectively, $F(1,15)<1$ ]. These results suggest that position information is as readily available from dichoptically masked word stimuli as is graphemic word information.

\section{Data Set 2}

The apparatus for collection of Data Sets 2 and 3 employed a computer-controlled monochrome (green) display, which was adapted for dichoptic presentation in the fashion described by Cheesman and Merikle (1986). The subject viewed the display through a $65-\mathrm{cm}$ long translucent white Plexiglas tube, which was rectangular in cross-section $(19.5 \mathrm{~cm}$ high $\times 26.0 \mathrm{~cm}$ wide) and contained a vertical divider that split the display into leftand right-eye fields. Two sets of rotating prisms at the subject's end of this viewing tube could be adjusted to permit superimposition (binocular fusion) of the left- and right-eye fields. The device could function as an $n$-field tachistoscope, the major restriction on which was that display durations could only be in multiples of the $20-\mathrm{msec}(50-\mathrm{Hz})$ display rewrite rate. This device allowed wider variation of brightnesses than had been possible on the Gerbrands tachistoscope. Except as noted, displays were composed of standard uppercase characters from the IBM PC text font. Individual letters occupied spaces that were $.25 \mathrm{~cm}$ wide $\times .50 \mathrm{~cm}$ high (approximately $.22^{\circ} \times .44^{\circ}$ at the $65-\mathrm{cm}$ viewing distance).

Initial exploration with this apparatus indicated that relative brightnesses of the dominant- and nondominant-eye fields more powerfully determined masking effectiveness than did exposure duration. Stimulus luminances for the display were measured from uniformly illuminated and darkened areas of the screen. In the normally lighted room in which the data were collected, the illumination of dark screen areas was measured at an average of $39.0 \mathrm{~cd} / \mathrm{m}^{2}$. The mask was presented at the high-intensity level of the display, for which illuminated areas were measured at an average of $136.5 \mathrm{~cd} / \mathrm{m}^{2}$. (The mask occupied 3 rows $\times$ 15 columns, and consisted of random orderings of the consonants $B, Q, W$, and $Z$.) Word stimuli were presented at the normalintensity level, for which illuminated areas were measured at an average of $67.8 \mathrm{~cd} / \mathrm{m}^{2}$.

Data Set 2 was provided by 6 subjects who were recruited for the purpose of comparing the difficulties of six perceptual tasks. Each subject participated in five daily sessions, providing 50 trials per day on each of six tasks, each presented at two durations (60 and $100 \mathrm{msec}$ ), with simultaneous dichoptic masking for all trials. The six tasks were (1) position discrimination with the stimuli LEFT and RIGHT; (2) position discrimination with evaluatively polarized word stimuli; (3) presence/absence discrimination with the stimuli LEFT and RIGHT; (4) presence/absence discrimination with evaluatively polarized word stimuli; (5) letter discrimination (four-alternative forcedchoice discrimination on each trial among XXXXX, SSSSS, $\mathrm{OOOOO}$, and $\mathrm{HHHHH}$ ); and (6) evaluatively polarized word discrimination (four-alternative forced-choice discrimination on each trial among HAPPY, SAD, GRIEF, and JOY).
In general, performance accuracy was greater than expected. All subjects showed substantial improvement within the first session, then relatively stable performance thereafter. Such improvements had not typically been observed when the Gerbrands tachistoscope was used as the display device. Presumably, the faster pace of trials and the larger number of trials permitted by the computerized apparatus permitted the improvements in performance. Nevertheless, all subjects had substantial error rates at all tasks, permitting comparisons of task difficulty.

The main interest was in the comparison between position discrimination and presence/absence judgments. Combined over the two stimulus durations, the position-discrimination task was easier than the presence/absence judgment for 3 of the 6 subjects with LEFT and RIGHT as stimuli (6-subject mean $d^{\prime} \mathbf{s}=$ 2.35 and 2.07 , respectively), and also for 3 of the 6 subjects with polarized word stimuli (mean $d^{\prime} s=1.98$ and 1.99 ). For the specific comparison corresponding to the two types of masked stimuli used in the three main experiments, 5 of the 6 subjects performed more accurately on position discrimination with LEFT and RIGHT (mean $d^{\prime}=2.35$ ) than on presence/absence discrimination with polarized word stimuli (mean $d^{\prime}=1.99$ ). With the small number of subjects, however, these differences between average performance levels do not approach statistical significance.

These results suggest that position discrimination and presence/absence judgments are approximately equally difficult. Accordingly, the thresholds set in the main experiments using the position-discrimination task should be approximately the same as ones that would have been set by use of a forced-choice presence/absence judgment task.

All 6 subjects who contributed to Data Set 2 showed poorer performance on both letter discrimination (mean $d^{\prime}=0.95$ ) and word discrimination (mean $d^{\prime}=0.97$ ) than on position discrimination and presence/absence judgments (mean $d^{\prime}=2.10$ ). This greater difficulty of letter and word discrimination than of position discrimination indicates that the conditions of the main experiments (which rendered position information undetectable) should be ones that did not permit letter or word information to be discriminable.

\section{Data Set 3}

The final set of data focused more specifically on a comparison of accuracy on position discrimination, using LEFT and RIGHT as stimuli, and presence/absence discrimination, using evaluatively polarized word stimuli. (The evaluatively polarized word stimuli were the priming stimuli from Experiments 1 and 2.) The procedure used for Data Set 2 was modified by reducing the brightness of stimuli to the nondominant eye (to lower accuracy levels) while also using a longer presentation duration $(300 \mathrm{msec})$. The intensity of illuminated areas in the nondominant-eye field was measured at an average of $58.0 \mathrm{~cd} / \mathrm{m}^{2}$. Also, the mask was changed to a set of graphics characters that looked like small boxes, and was given apparent motion by using a one-character position shift of the middle row of the mask every 100 msec. The choice of this masking procedure, which was discovered to be very effective, was based on a report that motion is an especially effective cue for capturing visual dominance in a binocularly rivalrous display (Duensing \& Miller, 1979).

Fifteen subjects provided data for 100 trials on each of the two tasks. Mean performance levels were very similar for po- 
sition discrimination (mean $d^{\prime}=0.28$ ) and presence/absence judgments [mean $d^{\prime}=0.43, F(1,14)=2.22$, n.s.]. Furthermore, performance levels on the two tasks were highly correlated $[r(13)=.95]$. The combination of similar levels of performance and high intercorrelation supports the conclusion that the two tasks are approximately interchangeable.

\section{Conclusion}

Three findings from the supplementary data sets provide some assurance that the position-discrimination task used in Experiments 1,2 , and 3 was appropriate for determining whether masked words were detectable to subjects. First, position discrimination and presence/absence judgments were very similar in difficulty in the two data sets (Sets 2 and 3) in which they were directly compared. Second, the very high correlation $(r=.95)$ between performance on position discrimination and performance on presence/absence judgments in Data Set 3 indicates that the two tasks measure essentially the same percepual ability. Third, the finding in Data Set 2 that all subjects performed better at position discrimination than at either letter or word discrimination further strengthens the conclusion that subjects in the main experiments should not have been able to detect letter or word information.

(Manuscript seceived March 16, 1987; revision accepted for publication March 21, 1988.) 